\title{
Uso de fármacos durante el período de gestación en embarazadas de Buenos Aires, Argentina
}

\author{
Taking medicine during pregnancy in females living in Buenos Aires, \\ Argentina \\ Gustavo Horacio Marín ${ }^{1}$, Martín Cañas ${ }^{1,2}$, Cecilia $\operatorname{Homar}^{1}$, Corina Aimetta $^{3}$ y \\ Jimena Orchuela ${ }^{3}$
}

1 Facultad de Ciencias Médicas, Universidad Nacional de La Plata, Argentina. farmacomarin@yahoo.com.ar, macanas@netverk.com.ar, cecihom@gmail.com

2 Federación Médica de la Provincia de Buenos Aires (FEMEBA), La Plata, Argentina.

3 Facultad de Humanidades, Universidad Nacional de La Plata, Argentina. jiorchu@hotmail.com, caimetta@gmail.com

Recibido 1 Febrero 2010/Enviado para Modificación 21 Noviembre 2010/Aceptado 18 Diciembre 2010

\section{RESUMEN}

Objetivo Describir el uso de medicamentos durante el período de gestación en embarazadas de Buenos Aires Argentina.

Métodos Una muestra al azar obtenida entre mujeres que tuvieron su parto durante el año 2008 fueron encuestadas y entrevistadas respecto a datos generales, aspectos relacionados con la atención de su embarazo, características del parto y uso de fármacos durante la gestación. Las historias clínicas de las pacientes fueron revisadas para confirmar la información obtenida. Los medicamentos utilizados fueron clasificados en riesgos bajo, mediano o alto para el feto. Después del parto se realizó un examen físico a todos los recién nacidos, para detectar algún defecto congénito.

Resultados Fueron incluidas en el estudio 1338 mujeres, de las cuales 90,6 \% ingirió al menos 1 fármaco durante el período de gestación mientras que el 81,9\% utilizó medicamentos aparte del ácido fólico y hierro indicados habitualmente como profilaxis. El uso promedio de fármacos utilizado fue de 2 por paciente. Hierro $(71,8 \%)$, folato $(40,3 \%)$ antimicrobianos (40,9\%); analgésicos (38,5\%), antieméticos (30,8\%) y vitaminas $(19,7 \%)$ fueron los medicamentos más usados. El análisis de regresión logística sobre el uso de medicamentos mostró un incremento del uso en mujeres mayores, con un alto nivel educativo y socioeconómico. La automedicación fue del $32,3 \%$. El uso de medicamentos considerados de alto riesgo por FDA fue del 21,5\%. $\mathrm{La}$ incidencia de malformaciones detectadas fue del $0,6 \%$.

Conclusión Nueve de cada 10 mujeres ingirieron al menos a un fármaco durante su embarazo. Con el fin de evitar la exposición innecesaria a riesgos potenciales, el uso de medicamentos durante el período de gestación debe restringirse estrictamente a una necesidad cierta y con la mayor relación beneficio/riesgo.

Palabras Clave: Embarazo, utilización de medicamentos, riesgo (fuente: DeCS, BIREME). 


\section{ABSTRACT}

Objective Describing how medicine was taken by pregnant females living in Buenos Aires, Argentina, during 2008.

Methods A random sample of females who had delivered during 2008 was surveyed and interviewed regarding pertinent general data, the health care they had received during pregnancy and characteristics concerning their deliveries. Information related to antenatal care included maternal reports concerning the use of medicine during pregnancy; medicine was classified as having low, medium or high risk for the foetus. Prescriptions were also analysed. A physical examination of all newborns was performed after delivery to detect any birth defect.

Results $90.6 \%$ of the 1,338 women included had taken at least one medicine/drug during their pregnancy and $81.9 \%$ had taken medicines apart from folate-iron (usually indicted as prophylaxis). Average medicine/drug use was two per pregnant female. Iron $(71.8 \%)$, folic acid (40.3\%), antibiotics (40.9\%), analgesics, antiemetics (30.8\%) and vitamins $(19.7 \%)$ were the medicines which were most used throughout pregnancy. Logistic regression analysis of medicine use showed increased usage risk for older women, those having a higher education level or those having higher socioeconomic status. Self-medication was observed in $32.3 \%$ of the sample; $21.5 \%$ of the medicines consumed were considered as being high risk medicines by the US FDA. The incidence of malformations detected was $0.6 \%$.

Conclusion 9 out of 10 females had used at least one drug/medicine during their pregnancy. To avoid unnecessary exposure to potential risk during pregnancy medicine use should be strictly related to patient's health needs, in line with the best benefit/risk ratio.

Key Words: Pregnancy, drug use, risk (source: MeSH, NLM).

a prescripción de medicamentos en el embarazo es muy frecuente. Casi todas las mujeres gestantes están expuestas a algún tipo de medicamento durante el embarazo. Pero aunque la mayoría de las mujeres embarazadas consumen medicamentos por prescripción o de venta libre regularmente, se dispone de pocos datos sobre su eficacia y seguridad en este subgrupo de pacientes (1).

Al momento de prescribir o dispensar un fármaco a una paciente embarazada, se está frente a un desafío único: hay dos pacientes involucrados mediante una sola práctica. Debido a ello es necesario realizar un minucioso balance beneficio-riesgo y tener presente que cualquier medicamento suministrado puede tener efectos indeseables sobre el embrión o el feto desde la concepción hasta el nacimiento (2).

La información sobre seguridad de los nuevos medicamentos que ingresan al mercado es incompleta por cuestiones inherentes al diseño de los ensayos clínicos controlados. Esta carencia de información se agudiza en las mujeres 
embarazadas ya que por cuestiones éticas casi no se realizan este tipo de estudios y solo unos pocos medicamentos han sido probados específicamente para la seguridad y eficacia durante el embarazo (3) La información de los efectos adversos y tóxicos de los medicamentos durante la gesta proviene entonces principalmente de la extrapolación de los datos de estudios preclínicos y estudios observacionales (4). Es por ello que frecuentemente la seguridad de un medicamento para las madres y sus fetos no se puede determinar hasta que se utilizan ampliamente.

Ante la falta de esta información muchas mujeres rehúsan a tomar o retrasan el comienzo del tratamiento con medicamentos que son recomendados durante la gestación y no ameritan discusión alguna. Es el caso del ácido fólico, además de reducir la ocurrencia de anemia megaloblástica de la embarazada, reduce el riesgo de malformaciones del tubo neural (5).

Por ello, el empleo de medicamentos es imprescindible en muchas ocasiones para la culminación adecuada de la gestación, y en estos casos debe favorecerse su uso más racional.

Estimaciones internacionales establecen que un 2-3\% de los recién nacidos poseen anomalías congénitas y de estas un 2-5\% son atribuidas a medicamentos y pueden prevenirse en la mayor parte de los casos (6-8).

La evaluación de los potenciales riesgos asociados al uso de un medicamento durante el embarazo es dificultoso, ya que además de potencial intrínseco para producir malformaciones o trastornos durante la gestación y el parto, debe también tenerse en cuenta el trimestre del embarazo en que se utiliza, la dosis y duración del tratamiento y la patología materna que origina su uso.

Para resumir, debido a los escasos datos existentes sobre la seguridad de los medicamentos durante el embarazo, varios países se han creado sistemas de clasificación de riesgo para informar y advertir a su población sobre el consumo de fármacos durante el período gestacional. Sin embargo, estas clasificaciones han dado lugar a afirmaciones ambiguas que a menudo son difíciles de interpretar y utilizar con precisión por parte de los pacientes. Las más utilizadas son las clasificaciones de la FDA de USA y la clasificación Australiana $(9,10)$.

Estudios realizados a nivel internacional indican que alrededor del $60 \%$ al $80 \%$ de las mujeres consumen medicamentos durante el embarazo. Los más frecuentes son analgésicos, antieméticos, vitaminas, cafeína, antibióticos, tabaco y alcohol $(11,12)$. 
Resulta importante establecer estudios actualizados a nivel regional, para determinar cual es la realidad actual en los diferentes países latinoamericanos.

Con el objetivo de determinar la prevalencia de uso de medicamentos en la provincia de Buenos Aires, lugar de residencia del $40 \%$ de la población de Argentina, se realizó el presente trabajo.

\section{MÉTODOS}

Se realizó un estudio descriptivo y prospectivo de utilización de medicamentos, en el período Diciembre 2007 a Diciembre de 2008. Se incluyeron embarazadas captadas en los Hospitales Públicos pertenecientes a 20 distritos de la Provincia de Buenos Aires.

El instrumento para la recolección de datos fue una encuesta semiestructurada que se realizó a las madres durante su internación para su parto o cesárea.

Alumnas del último año de la Carrera de Obstetricia, fueron capacitadas durante un período de 3 meses en la metodología de encuesta y definiciones operativas para llevar adelante el estudio.

La información explorada incluyó datos generales, datos socioeconómicos, laborales y nivel educativo de la paciente; datos de la gesta, información sobre los medicamentos consumidos, motivo de su indicación, quién fue el prescriptor, momento de la prescripción y duración del tratamiento.

Los medicamentos consumidos fueron clasificados según las cinco categorías de la FDA, identificadas mediante las letras A, B, C, D y X. La definición de la categoría A significa que no hay riesgo, y en la categoría $B$ es poco probable que existan riesgos. Para los medicamentos en la categoría $\mathrm{C}$ no hay datos adecuados. La definición de la categoría D es la siguiente: "existen evidencias de riesgo en los fetos humanos, pero los beneficios de su uso en mujeres embarazadas pueden ser aceptables a pesar del riesgo" (por ejemplo, en una situación potencialmente mortal). Por último, los fármacos clasificados dentro de la X están "contraindicados en mujeres que están o pueden estar embarazadas" (9).

La encuesta se complementó con una entrevista a la embarazada, con el fin de precisar algún aspecto de la encuesta que fuese pausible de aclaración. Luego del parto, se realizó un examen físico a todos los recién nacidos en los 
primeros tres días de vida, con el objetivo de detectar la presencia de algún defecto congénito, como disrupciones, malformaciones, o deformaciones en los mismos.

\section{RESULTADOS}

Se encuestaron 1338 pacientes en el período descripto. Los datos generales de las pacientes encuestadas mostraron que el $16,5 \%$ de las gestantes cursaba su primer embarazo y 10,9\% refirió haber tenido 5 o mas gestaciones. Un 32,8 \% tuvo 1 solo parto previo al momento de ser encuestada, 26,3\% 2 partos, 19,9\% 3 partos, 9,7 \% 4 partos, $6,6 \% 5$ partos y $4,7 \%$ mas de 6 partos.

En el $64,6 \%$ de los casos, el nacimiento fue producto de partos mientras que en el $35,4 \%$ se registraron cesáreas. Un 46,2\% refirió no haber tenido abortos, un $17,5 \%$ refirió 1 a 2 abortos previos, $4,1 \%$ tuvo 3 o mas abortos; y un 32,5\% de las encuestadas se negó a contestar esa pregunta.

El 11,4\% de las embarazadas encuestadas eran extranjeras (Bolivia 3\%, China $0,3 \%$, Ecuador $0,3 \%$, Paraguay $5 \%$, Perú $1,5 \%$, Uruguay $0,3 \%$ ), y $5.4 \%$ noeran bonaerenses sino que provenían de otras provincias Argentinas.

Solo el 8,9 \% de las encuestadas poseía obra social. El estado civil de las encuestadas mostró un $20,4 \%$ de solteras, $15,7 \%$ de casadas, $63,6 \%$ en concubinato, $\mathrm{y}$ viudas en un $0,15 \%$.

El nivel educativo de las encuestadas fue el siguiente: 57,8 \% completó su instrucción primaria, $37,2 \%$ completó su nivel secundario, $1,5 \%$ tuvo nivel terciario, $1,2 \%$ tenía un nivel Universitario y un 2,7 \% no había pasado por el sistema educativo.

En promedio de controles realizados a las embarazadas encuestadas fue de 5,1 . Un $17,2 \%$ no tuvo controles de embarazo durante el periodo de gestación, $9,8 \%$ tuvo 1 control, $12,3 \% 2$ controles, $10,4 \% 3$ controles, $10,6 \% 4$ controles, $13,9 \% 5$ controles, $15,1 \% 6$ controles; y 10,7 \% 7 o mas controles.

No se hallaron diferencias significativas entre la nacionalidad o la procedencia de la embarazada y el número de controles realizado. Sin embargo, comparando el nivel educativo alcanzado en relación al numero de controles, se observó que aquellas pacientes sin instrucción o con solo con escolaridad primaria, realizaron menos controles en promedio que las que poseen mayor instrucción (3,2 vs 6,9; $\mathrm{p}=0,01)$. 
Los controles se efectuaron en un 58,9 \% de los casos en los Centros de Atención Primaria de la Salud, y un 31,1\% en los Hospitales Públicos. Un $75,8 \%$ refirió no haber tenido inconvenientes en la accesibilidad a realizar los controles prenatales. Aquellas encuestadas que manifestaron dificultad en el acceso, refirieron como los mayores inconvenientes para efectivizar las consultas a los costos de traslado, el tiempo de espera o la distancia a recorrer desde su hogar hasta el sitio de control.

El control del embarazo fue realizado en un $60,1 \%$ por obstétrica, 35,7\% por médico toco-ginecólogo y en un $4,3 \%$ por un medico generalista o de familia.

El 92,5\% de los recién nacidos pesaron más de 2500 gramos mientras el 7,5 \% tuvieron un peso menor a dicha cifra. Cuando se analizó el grado de instrucción de la madre en relación al bajo peso, se observó que un 8,1 \% de aquellas madres sin instrucción o solo con instrucción primaria, tuvieron un niño de bajo peso, mientras que en aquellas con un nivel secundario o terciario este porcentaje se redujo al 4,9\% ( $\mathrm{p}=0,03)$. En cuanto al origen de la madre, no hubo diferencias significativas entre aquellas extranjeras y las argentinas (el porcentaje de recién nacidos menor a 2500 gramos fue de 6,4 vs 7,3 respectivamente; $\mathrm{p}=0,4)$.

Con respecto al consumo de fármacos, El porcentaje total de gestantes que consumió medicamentos durante su embarazo fue del 90,6 \%. Excluyendo el ácido fólico y el hierro, este porcentaje fue del 81,9\%.

El consumo fue resultado de una prescripción profesional (médico u odontólogo) en el 66,7 \%, mientras que fue producto de automedicación (sin prescripción médica) en el 32,3\%.

El período en el cual se realizaron la mayor cantidad de prescripciones o consumo de medicamentos fueron en el primer y tercer trimestre de la gestación (33,9\% y 38,7 \% respectivamente), siendo menor el consumo durante el segundo trimestre $(27,4 \%)$. Si se excluyen el folato y el hierro, igualmente es en el primer y último período del embarazo en los cuales se registró el mayor consumo de fármacos, $30,4 \%$ y $42,7 \%$ versus un $26,9 \%$ de consumo de medicamentos en el segundo trimestre.

Las causas que con mayor frecuencia ameritaron una prescripción o la toma de medicamentos fueron profilaxis de defectos del tubo neural $(39,9 \%)$, 
profilaxis de anemia $(62,2 \%)$, tratamiento de anemia $(60,1 \%)$, trastornos gastrointestinales $(43,1 \%)$, infecciones $(41,7 \%)$, profilaxis Estreptococo Beta Hemolítico $(15,3 \%)$, dolor $(27,7 \%)$, profilaxis de fenómenos trombóticos o síndrome anti-fosfolipídico $(1,6 \%)$, profilaxis de tétanos $(63,3 \%)$, profilaxis Enfermedad Hemolítica del Recién Nacido por incompatibilidad Rh (2,8 \%), hipertensión (3,5\%), DBT gestacional $(1,8 \%)$, amenaza de parto prematuro o contracciones de pre-término $(16,9 \%)$, motivos inaceptables o irracionales: $14,9 \%$.

Los grupos farmacológicos mayormente consumidos fueron: hierro $(71,8 \%)$, vacuna antitetánica $(63,3 \%)$, antimicrobianos $(40,9 \%)$; folatos $(40,3 \%)$, analgésicos y antipiréticos (38,5\%); antieméticos (30,8\%), suplementos vitamínicos (complejos polivitamínicos 19,7 \%); antimicóticos (18,7 \%), antiprotozoarios (anti-tricomoniasicos) $(15,8 \%)$; laxantes o anti-diarreicos $(11,9 \%)$, ansiolíticos $(6.7 \%)$, corticoides $(5,7 \%)$, antihipertensivos $(5,8 \%)$, broncodilatadores $(4,7 \%)$, antihistamínicos $(4,6 \%)$ yAZT $(0,4 \%)$. El riesgo que conllevan los fármacos consumidos según la FDA fue de $3,2 \%$ para medicamentos considerados como riesgo $\mathrm{X}, 7,2 \%$ con riesgo $\mathrm{D}, \mathrm{y} 11,1 \%$ con riesgo $\mathrm{C}$.

El consumo de tabaco durante la gestación se dio en el $26,1 \%$ de las encuestadas, mientras que 59,6\% de las encuestadas admitió haber consumir alcohol durante el período de gestación (en un 23,8 \% el consumo de alcohol fue de al menos 30 gramos semanales). El consumo de sustancias adictivas (marihuana y cocaína) fue de $3,2 \%$ y $0,4 \%$ respectivamente.

La mayor tasa de consumo se objetivó con mayor frecuencia en las madres mayores a 30 años $(62,4 \%$ vs $37,3 \%$ p 0,01), con un nivel socioeconómico superior $(54,1 \%$ vs $35,9 \%$ p 0,01$)$, y en aquellas cuyo trabajo se encuentra fuera del hogar $(44,4 \%$ vs $34,6 \%$; $=0,03)$.

El consumo de ácido fólico en las primeras semanas de la gesta (recomendado por las autoridades sanitarias Nacionales y Provinciales), solo se cumplió en el 39,9 \% de los casos. Se consideró para ello, el período comprendido entre 3 semanas antes del diagnóstico de embarazo, hasta las 12 semanas posterior a la fecha de última menstruación.

La incidencia de malformaciones detectada fue del $0,6 \%$ y no se observaron diferencias estadísticamente significativas entre los grupos que consumieron medicamentos versus aquellos que no consumieron $(0,7$ vs 0,5$)$ 


\section{DISCUSIÓN}

El consumo de fármacos durante el período de gestación es elevado. En muchos casos estos fármacos se encuentran rutinariamente indicados como la vacuna antitetánica. Asimismo, teniendo en cuenta que la prevalencia de anemia ferropénica asociada al embarazo es elevada, resulta aceptable la elevada frecuencia con la que se observa el uso en forma terapéutica o profiláctica de administración de sales de hierro (13). Sin embargo, aún excluyendo la vacunación o el consumo de hierro y folato habitualmente indicados en el embarazo, más del $80 \%$ de las gestantes encuestadas admitió haber consumido medicamentos durante la gesta actual, siendo $2 / 3$ de ellos producto de las prescripciones médicas u odontológicas y $1 / 3$ de la automedicación. Los motivos más frecuentes que originaron la automedicación fueron, fiebre, dolores e infecciones de vías aéreas superiores. Sin bien la evidencia sobre el riesgo de aparición de malformaciones congénitas asociada al uso de AINES es escasa, en la mayoría de los casos, la ingesta de de estos fármacos en las situaciones analizadas podría haberse evitado. Cabe destacar además que el uso de los AINES no está recomendado después de la semana 30, ya que disminuyen la contractilidad uterina, con posibilidades de prolongar el parto y producir cierre prematuro del ductus arterioso.

Si se considera que existe asociación demostrada de ingesta de medicamentos durante el embarazo con ciertas malformaciones congénitas (14) y que el $68 \%$ del consumo consiste en medicamentos con moderado a alto riesgo potencial, es preocupante que exista una elevada ingesta como la descripta tanto en el presente estudio como en estudios similares $(15,16)$. Aún siendo estos fármacos prescriptos por profesionales de la salud, los mismos conllevaban riesgos en un $76 \%$. (16)

Resulta necesaria una actualización de las clasificaciones de riesgo potencial actualmente vigentes, debido a que: se incorporan continuamente nuevos medicamentos en el mercado, la disponibilidad regional de diferentes medicamentos es variable, algunos medicamentos cambian de categoría según el trimestre que se considere y en función del aporte de nuevos conocimientos sobre su riesgo potencial.

Al respecto, es pertinente comentar un estudio similar al realizado en el presente trabajo, que comparó y analizó la coherencia entre los criterios para la clasificación de riesgo de los medicamentos usados durante el embarazo, incluida en 3 sistemas de clasificación internacional de riesgo ampliamente 
utilizados (EE.UU, Australia y Suecia). Se encontraron diferencias en la distribución de medicamentos para diferentes categorías de factores de riesgo. Sólo el 61 (26\%) de los 236 medicamentos comunes a todos los 3 sistemas fueron colocados en la misma categoría de los factores de riesgo. Los autores concluyeron que estas diferencias pueden ser fuente de gran confusión entre los usuarios de los sistemas de clasificación, así como para aquellos que requieren información sobre el riesgo del consumo de medicamentos durante el embarazo, y puede limitar la utilidad y la fiabilidad de los sistemas de clasificación de riesgo (17).

Desde el año 2001, en países como los EE.UU. se llevan a cabo nuevos estudios en el marco de un programa denominado Programa de Evaluación del Riesgo por Exposición a Medicación en el Embarazo. Dicho programa extrae datos de 11 sitios de investigación que cuentan con información sobre la atención médica de alrededor de 1 millón de nacimientos (18).

En el presente trabajo se encontró un elevado porcentaje de embarazadas que consumen algún tipo de medicamentos durante su período de gestación. Si bien se ha objetivado una baja prevalencia de malformaciones asociadas al uso de medicamentos durante la gesta, esas malformaciones o disrupciones acompañarán a individuo nacido durante toda su vida y marcarán al mismo en lo personal e impactarán en el sistema sanitario, social, y económico de un país (19). Es deseable pues, que se pueda reducir el consumo de medicamentos al mínimo indispensable durante el embarazo, es decir solo a la profilaxis de defectos del tubo neural, a la profilaxis de anemia o al tratamiento de enfermedades previas o que debuten durante el embarazo. Resulta recomendable desalentar el uso sin la prescripción médica adecuada.

Las políticas públicas deben garantizar acciones de prevención primaria que tengan como objetivo principal evitar el uso indebido de medicamentos durante el período de embarazo

\section{REFERENCIAS}

1. Buhimschi $C$, Weiner C. Medications in Pregnancy and Lactation: Part 1. Teratology. Obstetrics \& Gynecology. 2009; 113 (1): 166-188.

2. Briggs GG, Freeman RK, Yaffe SJ. Drugs in Pregnancy and Lactation. 6th ed. Philadelphia: Lippincott Williams \& Wilkins; 2002.

3. Doering PL, Boothby LA, Cheok M. Review of pregnancy labelling of prescription drugs: Is the current system adequate to inform of risks? Am J Obstet Gynecol. 2002;187:333-9.

4. Koren G. Ethical framework for observational studies of medicinal drug exposure in pregnancy. Teratology. 2002;65:191-5. 
5. Galloway R, Dusch E, Elder L, Achadi E, Grajeda R, Hurtado E, et al. Women's perception of iron deficiency and anemia prevention and control in eight developing countries. Soc Sci \& Med 2002; 55(4):529-44.

6. Rubio Barbon S, García Fernández ML. Utilización de fármacos durante el embarazo y la lactancia: Farm Hosp. 1993; 17: 3-24.

7. Hays DP, Pagliaro LA Human Teratogens. In: Pagliaro LA, Pagliaro AM (eds). Problems in pediatric drug therapy. 2nd ed. Hamilton IL: Drug Intelligence Publications, Inc, 1987. pp. 51-69.

8. CADIME. [Internet] Medicamentos y embarazo. Monografía . Boletín Terapéutico Andaluz n.o 81995. Escuela Andaluza de Salud Pública. Disponible en: http://www.easp.es/web/ documentos/MBTA/00001174documento.pdf. Consultado: enero 21, 2010.

9. Briggs GG, Freeman RK, Yaffe SJ. Drugs in Pregnancy and Lactation: A Reference Guide to Fetal and Neonatal Risk. 5th ed. Baltimore, MD: Williams \& Wilkins; 1998.

10. ADEC [Internet]. Prescribing medicines in pregnancy -An Australian categorisation of risk of drug use in pregnancy 4th edition; 1999. Disponible en http://www.tga.gov.au/docs/ $\mathrm{pdf} / \mathrm{medpreg.pdf}$ Consultado: enero 21, 2010.

11. Koren G, Pastuszak A, Ito S. Drugs in pregnancy. N Engl J Med 1998;338:1128-37.

12. Estudio multicéntrico sobre el uso de medicamentos durante el embarazo en España (III). Los fármacos utilizados durante el primer trimestre de la gestación. Med Clin (Barc) 1991;96:52-7.

13. Galloway R, Dusch E, Elder L, Achadi E, Grajeda R, Hurtado E, et al. Women's perception of iron deficiency and anemia prevention and control in eight developing countries. Soc Sci \& Med 2002; 55(4):529-44.

14. Laporte JR, Diogenes E, Aragón C, Vidal X.Detección y cuantificación de reacciones adversas sobre el embrión y el feto; estudios y registros de malformaciones congénitas. Med Clin (Barc) 1986;36:31-5.

15. Grupo de trabajo DUP España. Estudio multicéntrico sobre el uso de medicamentos durante el embarazo en España (II). Los fármacos utilizados durante la gestación. Med Clin (Barc) 1991;96:11-5

16. Carmo TA, Nitrini SM. Prescrições de medicamentos para gestantes: um estudo farmacoepidemiológico. Cad Saúde Pública 2005; 20(4):1-22.

17. Addis A, Sharabi S, Bonati M. Risk classification systems for drug use during pregnancy: are they a reliable source of information? Drug Saf. 2000;23(3):245-53.

18. EEUU estudiará la seguridad de los fármacos durante el embarazo. Reuters Health; 31 de diciembre de 2009

19. Bánhidy F, Lowry RB, Czeizel AE. Risk and Benefit of Drug Use During Pregnancy. Int J Med Sci 2005; 2:100-106. 